\title{
Performance Analysis of WCDMA using Different Spreading Codes
}

\author{
Dr. P. Samundiswary and P.V.S.R.S Viswa Kalyan \\ Dept. of Electronics Engineering \\ Pondicherry University \\ Pondicherry, INDIA
}

\begin{abstract}
Recently, Wideband Code Division Multiple Access (WCDMA) technology has emerged as the most widely adopted third generation (3G) air interface. Conventionally, the Pseudo random Noise (PN) sequence and gold code sequence are used as spreading codes in WCDMA. However enhanced performance using these sequences is achieved by increasing the length of the sequence. In this paper, Bit Error Rate (BER) performance of WCDMA is determined and analyzed by considering various spreading codes of affordable spreading factor in the presence of Rayleigh channel with AWGN. The performance of WCDMA system using Walsh Code is also compared with other spreading sequences such as $\mathrm{PN}$ and gold sequence for various Spreading Factors (SFs). However, simulation results show that the BER of WCDMA can be improved drastically by using Walsh code.
\end{abstract}

\section{Keywords}

WCDMA, PN sequence, Gold sequence, Walsh code, BER, AWGN.

\section{INTRODUCTION}

Third generation systems are designed for multimedia communication which can be enhanced with high quality video. These systems provide services with high data rates for public and private networks. WCDMA technology is the most widely used third generation system which is spreading over a wide bandwidth by multiplying the user information with spreading sequence in WCDMA [1]. The various spreading codes with different spreading length are used to support high bit rate and mitigate the effect of inter-symbol interference (ISI) and narrowband interference in WCDMA. The main property of the spreading code is that they need to be orthogonal to each other. The most popular spreading codes are Hadamard codes, PN sequence, Gold sequence and Walsh code s etc.

The pseudorandom (PN) sequence is deterministically generated; however it is almost like random sequences to an observer. The PN sequence is usually implemented by using sequential logic circuits [2]. These binary sequences are shifted through the shift registers in retort to clock pulses, and the output of the various stages are logically combined and fed back as the input to the first stage. This shift register is called a linear PN sequence generator. Since there are exactly $2^{\mathrm{m}}-1$ nonzero state for an $m$-stage shift register, a sequence of $2^{\mathrm{m}}-1$ generated by a linear feedback register is called a maximal length sequence. It increases circuit complexity by increasing the length of PN sequence and a clock has to be used both at the transmitter and the receiver that is one of the biggest disadvantages.
Gold sequence is another type of sequence used in WCDMA techniques [3]. Gold codes are obtained from a modulo-2 addition of two maximum length sequences. Gold sequences are generated on the basis of preferred pair m-sequences. Gold sequences have balanced property as that of PN sequence. The gold sequence has inter-code interference.

To achieve enhanced performance of WCDMA, Walsh code is used as spreading code with less spreading factor. In this paper, performance of the WCDMA with different spreading codes like PN sequence, Gold sequence, and Walsh codes are analyzed and compared through simulation. The paper is organized as follows: Section 2 describes about the WCDMA system and its characteristics. Section 3 discusses about the results and conclusion is drawn in Section 4.

\section{WCDMA}

WCDMA is intended for wideband multimedia services in the wireless communication industry. This WCDMA technology is also chosen as the air interface for the Universal Mobile Telecommunication System (UMTS) [4]. The 3GPP (3G Partnership Project for wideband CDMA standards) body is developing WCDMA for both wide area mobile cellular coverage as well as indoor cordless type applications [5]. WCDMA requires a minimum bandwidth allocation of $5 \mathrm{MHz}$, which is an important distinction from the other generation standards [6]. Packet data rates up to $2 \mathrm{Mb} / \mathrm{s}$ per user in indoor or small-cell outdoor environments and at rates of up to 384 $\mathrm{Kbit} / \mathrm{s}$ in wide-area coverage is supported by WCDMA [7]. Therefore, high quality data, multimedia, streaming audio, steaming video and broadcast-type services are allowed by using WCDMA technology to consumers. High service flexibility like support of multiple parallel variable rate services on each connection and support of inter frequency hand over and hand over to GSM system [8-9] is provided by WCDMA system. WCDMA employs coherent detection on uplink and downlink with the usage of pilot symbols and supports multiple types of handoffs between different cells including soft handoff, softer handoff and hard handoff. The basic block diagram of WCDMA is shown in Figure 1.

The performance of WCDMA system is further improved by using spreading codes. Walsh codes with different spreading codes are used to achieve better BER performance in WCDMA system. Generally, Walsh codes are used in direct sequence and frequency hopping spread spectrum (such as IS95 , cdma2000 etc.). Walsh codes are orthogonal codes. They are generated by using the Hadamard matrix. From the corresponding Hadamard matrix, the Walsh codes are given by the rows [10]. For a set of Walsh codes of length $n$, there consists of $n$ rows to form an $n \times n$ Walsh code square matrix. As such, if two Walsh codes are correlated, the result is intelligible only if these two codes are the same. 


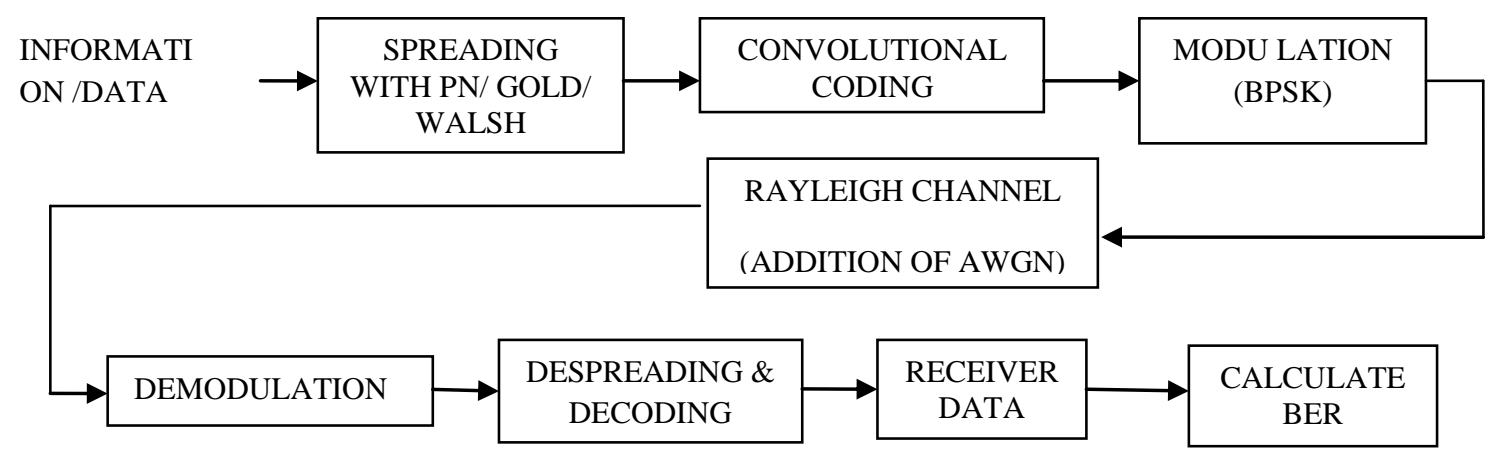

Fig 1: Basic Block Diagram of WCDMA

\section{SIMULATION RESULTS}

The WCDMA model is simulated for various spreading sequences such as PN, Gold and Walsh code in the presence of Rayleigh channel by considering 2 users and Binary Phase Shift Keying (BPSK) as modulation scheme. The simulation parameters used in the simulation are given in Table 1. Then the BER performance of WCDMA is calculated and compared by varying the length of above mentioned sequences. The BER is the number of bit received as errors divided by the total number of transmitted bits during a studied time interval [11].

$$
\text { BER }=\frac{\text { Errors }}{\text { Total Number of Errors }}
$$

Table 1. Simulation Parameters

\begin{tabular}{|l|l|}
\hline \multicolumn{1}{|c|}{ Parameter } & \multicolumn{1}{|c|}{ Value } \\
\hline Spreading codes & PN, Gold \& Walsh \\
\hline Spreading factor & $4,8,15,16,31,32,63$ \\
\hline No. of users & 2 \\
\hline Modulation technique & BPSK \\
\hline Channel & Rayleigh channel with AWGN \\
& \\
\hline
\end{tabular}

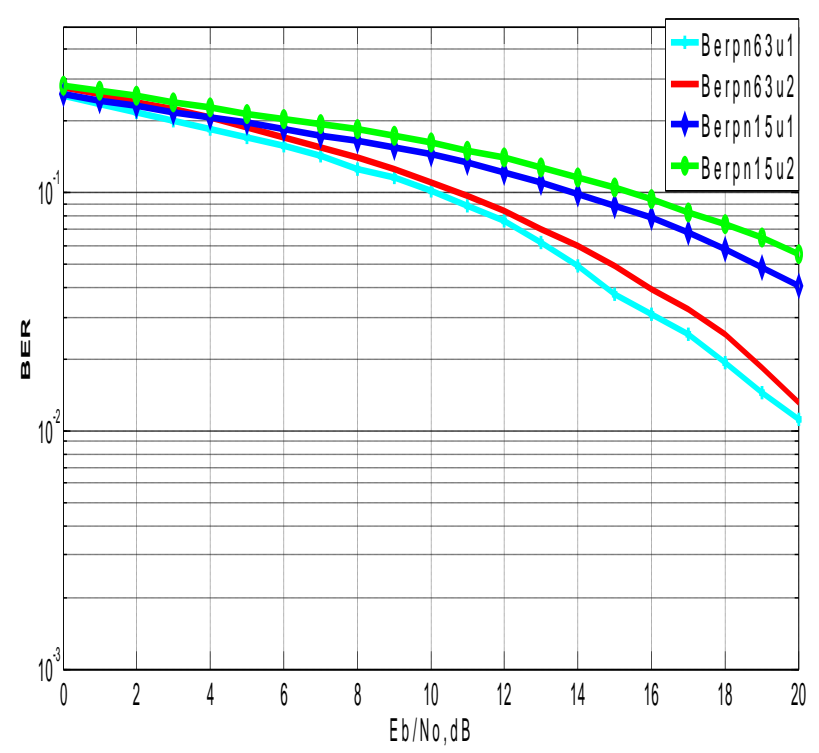

Fig 2: Comparison of PN sequences with SF of 15 and 63

From the Figure 2, it is observed that BER of WCDMA using PN sequence of spreading factor 63 is lesser than that of PN sequence of spreading factor 15 . The reason is that higher value of spreading factor reduces the error rate. It is inferred through the simulation result shown in Figure 3, gold sequence of SF 15 has higher BER than that of gold sequence of SF 31. Similarly, walsh Code with spreading factors of length 8 has lower BER compared to that of spreading factor of 4 which is illustrated through simulation result shown in Figure 4. 


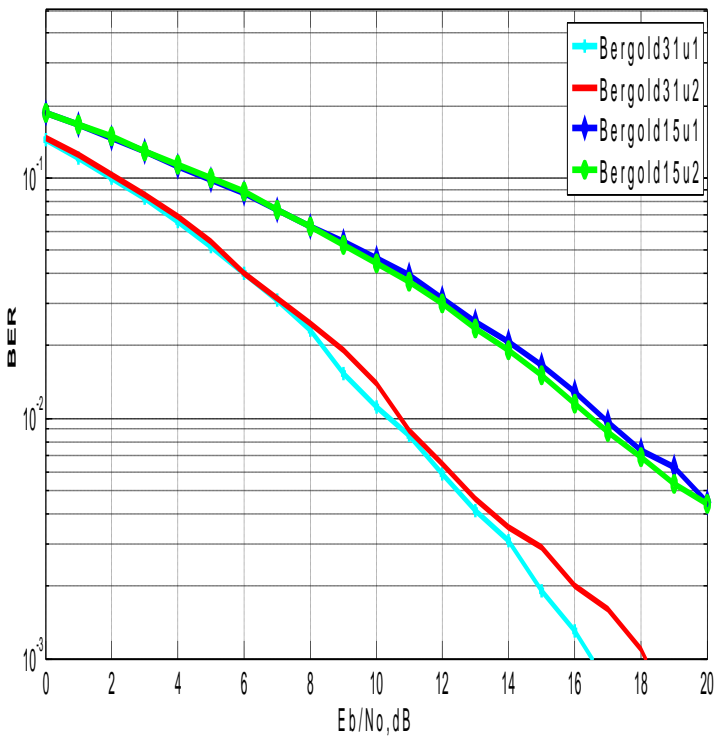

Fig 3: Comparison of Gold sequences with SF of 15 and 31

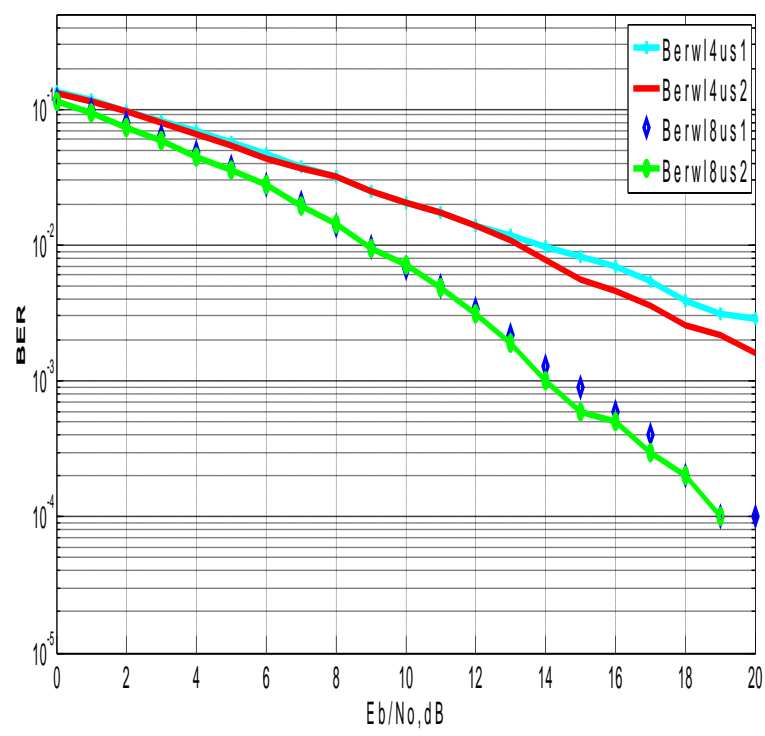

Fig 4: Comparison of Walsh sequences with SF of 4 and 8

The comparison of BER Vs $E_{b} / N_{o}$ for different spreading codes such as PN sequence, gold sequence and Walsh code with SF of 16 and 32 respectively are demonstrated in Figure 5 and Figure 6. It is also verified through the results shown in Figure 5 and Figure 6 that BER of WCDMA using Walsh sequence with spreading factor of 15 and 32 is approximately $10^{-4}$ at $E_{b} / N_{o}$ of $15 \mathrm{~dB}$ and $10^{-4}$ at $E_{b} / N_{o}$ of $13 \mathrm{~dB}$ respectively. Hence walsh code has better BER rate than that of Gold and $\mathrm{PN}$ sequences.

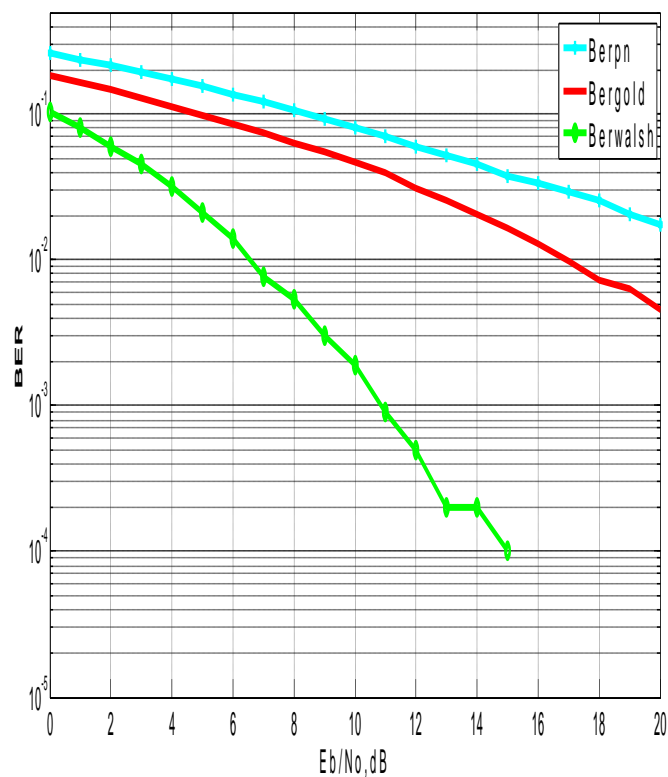

Fig 5: Comparison of various spreading codes with SF of 16

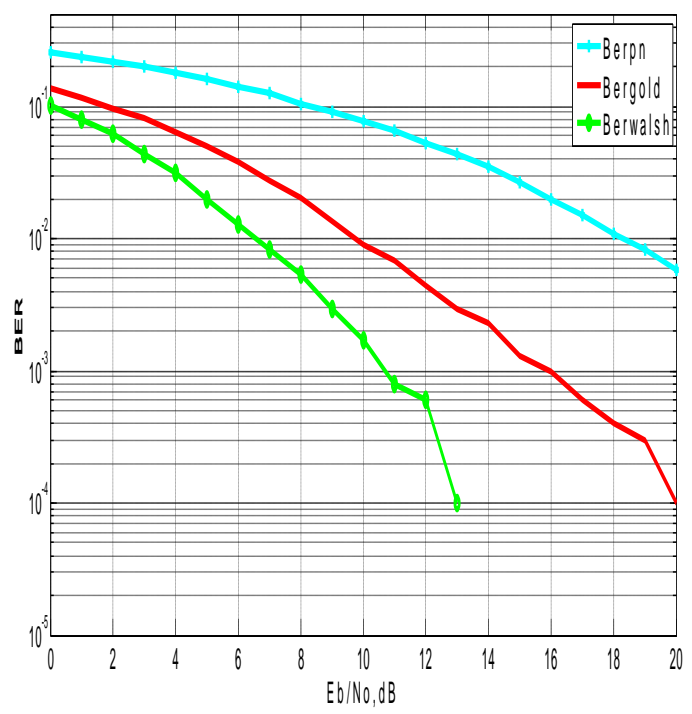

Fig 6: Comparison of various spreading codes of same $\mathrm{SF}=32$

\section{CONCLUSION}

The performance of WCDMA is analyzed by considering various spreading codes with different spreading factors and BPSK modulation in the presence of Rayleigh channel. It is concluded from the simulation results that BER of WCDMA by using Walsh code is lesser than that of other spreading codes (PN and gold sequence) for increased value of spreading factor. A Walsh based spreading code generator is chosen because of the easier implementation of its hardware module. These codes can be further simplified later by using FPGA design. The usage of memory is not only be reduced by using an LUT cascade for the Walsh-based spreading code generator, but a faster working system can be developed. 


\section{REFERENCES}

[1] Harri Holma and Antti Toskala, "WCDMA for UMTS, Radio Access For Third Generation Mobile Communications", John Wiley \& Sons, Ltd., Third Edition, September 2004.

[2] W. R. Braun, "PN acquisition and tracking performance in DS/CDMA systems with symbol-length spreading sequences," IEEE Transactions on Communication, vol. 45, pp. 1595-1601, December 1997.

[3] Yong-Hwan Lee and Seung-Jun Kim, "Sequence acquisition of DS-CDMA systems employing gold sequences", IEEE Transactions on Vehicular Technology, vol.49, no.6, pp.2397-2403, November 2000 .

[4] Erik Dahlman, Bjorn Gudmundson, Matts Nilsson, and Johan Skold, "UMTS/IMT-2000 Based on Wideband CDMA", IEEE Communications Magazine, vol. 36, pp. 70-80, September 1998.

[5] Askari, Mehdi, Saadat, Reza, Nakhkash, Mansour, "The Comparison of Various Code Assignment Schemes in Wideband CDMA", Proceedings of the International
Conference on Computer and Communication Engineering, May 13-15, 2008.

[6] Andrea Goldsmith, "Wireless Communication", Stanford University, 2004

[7] Tero Ojampera, Ramjee Prasad, "Wideband CDMA for Third Generation Mobile Communication: Universal Personal Communication”, Artech House, USA, 1998.

[8] Rappaport, Theodore, "Wireless Communications: Principles and Practice", Prentice Hall, Second Edition, 2001.

[9] Garg, V. K., Wireless Network Evolution: $2 \mathrm{G}$ to $3 \mathrm{G}$, Prentice Hall: Upper Saddle River, NJ, 2002.

[10] Falkowsky, B., Yan, Shixing, "Fixed Sign Walsh Transform and Its Iterative Hardware Architecture," IEEE International Symposium on Circuits and Systems, vol.1, pp.484-487, May 2005.

[11] Breed, G., "High Frequency Electronics, Bit Error Rate: Fundamental Concepts and Measurement Issues", Technical Report, Summit Technical Media, Copyright January 2003 